\title{
Antifungal Susceptibility Patterns of Aspergillus species isolated from patients with Pulmonary diseases in Iraq
}

\author{
Alaa Hani Al-Charrakh ${ }^{1 *}$, Zaidan Omran Al-Mamory², Kawther Ajwad Al-Malaky ${ }^{1}$ \\ ${ }^{1}$ Department of Microbiology, College of Medicine, Babylon University, Hilla, Iraq. \\ ${ }^{2}$ Department of Biology, College of Science for girls, Babylon University, Hilla, Iraq.
}

\section{ARTICLE INFO \\ Article history: \\ Received on: 12/06/2017 \\ Accepted on: 27/10/2017 \\ Available online: 30/05/2018}

\section{Key words:}

Aspergillus, Antifungal

Susceptibility, E-test, COPD,

pulmonary TB.

\begin{tabular}{l}
\hline ABSTRACT \\
\hline Aspergillus infections are among the most feared opportunistic infections in humans because they are capable of \\
causing several distinct pulmonary diseases. The aim of this study was to determine the prevalence of Aspergillus \\
species in patients with pulmonary pneumonia and susceptibility testing to the most commonly used antifungal agents. \\
A total of 311 specimens were collected from patients presented with the pulmonary complaint. Out of which, 139 \\
$(44.6 \%)$ yielded Aspergillus growth. Aspergillus isolates were obtained from Bronchoalveolar lavage and sputum. \\
Ten species of Aspergillus were identified, A. flavus, A. niger, and A. terreus were the most frequently isolated \\
species. Antifungal susceptibility of 10 different Aspergillus spp. isolates were performed using E-test. Voriconazole \\
and caspofungin were having full inhibitory activity $(100 \%)$ against tested Aspergillus spp., while some resistance \\
was observed against itraconazole and posaconazole $(50 \%$ and $75 \%$ respectively). Aspergillus was found to be the \\
predominant fungal pathogen isolated from patients with pulmonary diseases, notably from patients with COPD and \\
pulmonary TB.
\end{tabular}

\section{INTRODUCTION}

Aspergillus infections are among the most feared opportunistic infections in humans because they are capable of causing several distinct pulmonary diseases (Krel et al., 2014). Aspergillus spp. is the main causal agent for fungal respiratory infections in the critically ill patient comes in second place after the fungi from the order Mucorales (Garnacho-Montero et al., 2013).

The Aspergillus genus contains many species and these are ubiquitous in our environment. They do not form part of the normal flora. Their spores are regularly inhaled without harmful consequences, but some species, notably A. fumigatus, are able to cause a range of diseases, including 1-Allergic bronchopulmonary aspergillosis (ABPA), which is, as its name suggests, an allergic response to the presence of Aspergillus antigen in the lungs and occurs in patients with asthma. ABPA occurs in some $10 \%$ of cystic

\section{"Corresponding Author}

Alaa Hani Al-Charrakh, Department of Microbiology, College of Medicine, Babylon University, Hilla, Babylon Governorate, Iraq. E-mail:ahani67@gmail.com fibrosis patients, 2-Aspergilloma in patients with pre-existing lung cavities or chronic pulmonary disorders. Aspergillus colonizes a cavity and grows to produce a fungal ball, a mass of entangled hyphae, and 3-Disseminated disease in the immunosuppressed patient when the fungus spreads from the lungs (Goering et al., 2013).

Aspergilloma occurs when the conidia that been inhaled enter a pre-existing cavity, germinate, and produce abundant hyphae in the abnormal pulmonary space. Those patients with previous cavitary disease (e.g. tuberculosis, sarcoidosis, emphysema) are at risk (Brooks et al., 2014). Nowadays, Voriconazole is considered the first choice for treatment of aspergillosis; amphotericin B and echinocandins (mainly caspofungin) used to treat this infection as well (Walsh et al., 2008; Mikulska and Viscoli, 2011). An emerging constraint associated with Aspergillosis therapeutics is the increasing resistance against triazole that observed in $A$. fumigatus isolates (Denning et al., 2011; Snelders et al., 2011).

The aim of this study was to determine the prevalence of Aspergillus species in patients with pulmonary pneumonia, and antifungal susceptibility testing of these clinical fungal isolates by E-test technique. 


\section{MATERIALS AND METHODS}

\section{Study design and duration}

This cross-sectional study was designed to assess the occurrence of Aspergillus species in patients with pulmonary infections. Specimen collection and analysis was carried out for four months $\left(1^{\text {st }}\right.$ November 2015 to $30^{\text {th }}$ February 2016). Specimens collected from the specialized center of Chest and Pulmonary diseases in Hilla city/Iraq. Each specimen was cultured and examined macroscopically and microscopically.

\section{Study subjects}

Clinical specimens were obtained from patients (70 male and 69 female), presented with pulmonary symptoms, such as bronchiectasis, recurrent infections (with fever and malaise, dyspnea, anorexia, weight loss, and chest pain), chronic obstructive pulmonary disease (COPD), or a suspected case of lung infection. Mean age of the patients was $43.68 \pm 10$ years; with a wide-ranging age varied from 1 day to 90 years. The patients were diagnosed clinically by specialists and were tabulated according to their age, gender, and presence of Tuberculosis infection or another type of pulmonary disease.

\section{Ethical approval}

Verbal consent was taken from each patient before sampling. Investigative standards were rigidly preserved, primarily concerning confidentiality. Moreover, this study was undisclosed, the participation of patients was optional, and verbal consent was received before data uptake process was started.

\section{Specimen collection and mycological analysis}

A total of 311 specimens were collected from patients presented with the pulmonary complaint. Specimens included Bronchoalveolar lavage fluid (BAL) and sputum. The specimens were transported by screw-capped cups to the Microbiology lab and each specimen was inoculated by streaking on two general culture media (Sabouraud Dextrose agar-supplemented with chloramphenicol and Malt extract agar), and by pouring on third medium namely Potato dextrose agar, Then incubated at $25^{\circ} \mathrm{C}$ for 2-7 days (Forbes et al., 2014).

\section{Antifungal susceptibility testing}

Antifungal susceptibility by E-test method was done on non-supplemented Muller Hinton Agar (MHA) according to the method described in M51-A and M38-A2 CLSI documents. E-test gradient strips of Posaconazole (POS), itraconazole (ITC), voriconazole (VO), and caspofungin (CAS) were obtained from Liofilchem Lab, Italy. The concentration gradient for each drug ranged from 0.002 to $32 \mu \mathrm{g} / \mathrm{ml}$. The strips were stored frozen at $-20^{\circ} \mathrm{C}$ until they were used in the study. The E-test was performed by following the manufacturer's instructions. Each solidified medium was inoculated by dipping a nontoxic (latex-free) sterile swab into the respective undiluted stock inoculum suspension and evenly streaking it in three directions over the entire surface of a $150-\mathrm{mm}$ Petri plate containing $60 \mathrm{ml}$ of medium. The plates were incubated at $35^{\circ} \mathrm{C}$, and the MICs were read at $24 \mathrm{~h}$ or $48 \mathrm{~h}$ as the concentration of drug that elicited $100 \%$ inhibition of growth (posaconazole, itraconazole, voriconazole) or as the minimum effective concentration (MEC, caspofungin).

There was a tendency of an agar-based method to produce false susceptible results after 24 hours which can be explained, e.g., as being due to the shift of the interpretive categories in some Aspergillus strains when comparing reading after $24 \mathrm{~h}$ and $48 \mathrm{~h}$ (Buchta et al., 2008). Therefore, in case of $A$. terreus, the first reading at $24 \mathrm{~h}$ was considered while in the case of the remaining Aspergillus spp. the second reading was considered.

\section{RESULTS AND DISCUSSION}

Out of total 311 specimens collected, 139 (44.6\%) yielded Aspergillus growth while 49 (15.7\%) yielded no growth. Aspergillus isolates were obtained from two types of specimens; 17 isolates (12.23\%) obtained from BAL and 122 isolates $(87.7 \%)$ were obtained from sputum.

The 139 Aspergillus isolates were gathered from 70 male $(50.3 \%)$ and 69 female (49.6\%). Six patients with ongoing tuberculosis infection were all given positive Aspergillus isolates.

Ten species of Aspergillus were identified (Table 1). A. flavus, A. niger, and $A$. terreus were the most frequently isolated species followed by $A$. fumigatus in the fourth place $(51 \%, 48.9 \%$, $28.7 \%$, and $11.5 \%$ ), respectively.

Table 1: Aspergillus species isolated from Bronchoalveolar lavage fluid and sputum.

\begin{tabular}{rcc}
\hline Aspergillus species & No. of isolates & $\mathbf{\%}$ \\
\hline Aspergillus niger & 68 & 48.9 \\
Aspergillus flavus & 71 & 51 \\
Aspergillus fumigatus & 16 & 11.5 \\
Aspergillus terreus & 40 & 28.7 \\
Aspergillus oryzae & 1 & 0.71 \\
Aspergillus tumorous & 4 & 2.8 \\
Aspergillus candidus & 9 & 6.4 \\
Aspergillus tamari & 3 & 2.15 \\
Aspergillus parasiticus & 3 & 2.15 \\
Aspergillus versicolor & 3 & 2.15 \\
Total & $\mathbf{1 3 9}$ & $\mathbf{1 0 0 \%}$ \\
\hline
\end{tabular}

Some specimens had grown mixed Aspergillus culture (63 isolates (45.32) out of 139) therefore the total number of positive Aspergillus isolates $(\mathrm{N}=139)$ does not equal the total number of the individual isolates obtained $(\mathrm{N}=218)$. The creditability of direct microscopy in the diagnosis of fungal infection in sputum smears has been proven.

In a study, all of the $27(100 \%)$ samples that were positive for Aspergillus spp. by direct sputum microscopy and culture. In contrary, examination by direct microscopy failed to detect three samples, which were later found to be positive with culture. Direct microscopy is therefore 90\% sensitive in detecting Aspergillus spp. in sputum smears (Njunda et al., 2012). 
Aspergillus spp. may be responsible for important clinical events from saprophytic colonization of the airways to rapidly invasive and life-threatening disseminated diseases, depending on the host immune status and the presence of underlying lung disease (Latgé et al., 2001).

Several authors found that the most frequent species of Aspergillus associated with clinical disease cases were A. flavus, A. terreus, and A. niger followed by A. fumigatus (Malik et al., 2009; Shujat et al., 2014).

Another study showed that $28.08 \%$ of total 381 isolates were belonging to the genus Aspergillus. A. fumigatus was the one having highest percentage of frequency $(29.9 \%)$, followed by $A$. niger (28.9\%), as well as the isolated species $A$. flavus and $A$. terreus and $A$. nidulans $(18.7 \%, 12.14 \%, 2.8 \%)$, respectively (Aziz, 2014). It was found that the four most frequently isolated Aspergillus species as a causative agent in the pulmonary infections are A. fumigatus, A. flavus, A. niger, and $A$. terreus (Njunda et al., 2012; Barberan et al., 2012; Diba et al., 2007).

Various studies reported that $A$. fumigatus is more prevalent in the Western countries, while A. flavus is more prevalent in the Middle East and Asia as the leading cause of invasive aspergillosis of the lungs (Patterson et al., 2000; Groll and Kolve, 2004; Chamilos and Kontoyiannis, 2005; Singh and Paterson, 2005; Pfaller et al., 2006). However, it has been found that $A$. niger, A. nidulans, and A. ustus are only rarely isolated (Pfaller et al., 2006; Shao et al., 2007). A. niger was considered as a non-probable cause of pulmonary Aspergillosis.

A. niger isolates were mostly accounted as contamination (Patterson et al., 2000). This leaves us to consider A. flavus, and A. fumigatus as two most probable cause of pulmonary Aspergillosis.

In Aspergillus infections, the lung continues to represent the most frequently involved site. Pulmonary infection is a phenotypical representation of the interaction between lowered defense mechanisms in the host and the virulence of the fungus (Pagano et al., 2008).

This study result showed that patients with pulmonary diseases and patients with lower immune status are mainly at risk of infection by pathogenic Aspergillus spp. and the fact that pulmonary abnormalities are a predisposing factor for the fungal infection.

Results also found that six patients were presented with tuberculosis. These patients with TB were having a concomitant Aspergillosis infection (6/139, 4.31\%). Moreover, along with those with proven tuberculosis, almost two-thirds of the patients $(211 / 311,67.84 \%)$ have had a history of chronic bronchitis, pneumonia, cancer, undergone COPD attack or suffering from COPD during the time when the sample has been taken or have another form of the pulmonary disease. Others have a suspected case of pneumonia with or without immunocompromisation.

In a single study, nine $9(4.5 \%)$ patients infected with both infections (Tuberculosis and Aspergillosis). Among the nine 9 patients who were infected with Aspergillus sp. and TB, six 6 were infected with $A$. fumigatus, two 2 with $A$. niger and one 1 with $A$. flavus. None of the patients was infected with A. terreus (Njunda et al., 2012). Previous tuberculosis (either classical or atypical) was the most commonly identified primary underlying condition $(38 / 126,30.2 \%)$. The second common, primary underlying condition was ABPA (15/126, $11.9 \%$ ). However, all of the 232 underlying conditions identified for 126 Chronic Pulmonary Aspergillosis (CPA) cases, COPD/emphysema was the most common (42/126, $33.3 \%)$.

\section{Antifungal susceptibility testing}

Since fungal infections in hospitals are much less frequent than other microbial infections, susceptibility tests for fungi are not routinely performed in some diagnostic laboratories (Badiee et al., 2012). In the present study, ten 10 Aspergillus spp. isolates were tested by using E-test against four antifungal agents ITC, POS, O, and CAS).

The E-test was carried on non-supplemented MHA (Figure 1). Antifungal agents showed good activity against Aspergillus spp. except for some forms of resistance which have detected in some isolates even though the antifungals tested are not in use nationally and it was the first time the fungus exposing to them (Table 2). A group of authors mentioned that there was no difference in MIC or MEC values at $24 \mathrm{hr}$ and $48 \mathrm{hr}$, therefore we suggest E-test be done on non-supplemented MHA in place of RPMI 1640 agar with 2\% glucose and MOPS, which is also the common media available in most of the laboratories (Gupta et al., 2015).

In the present study, resistance to ITC was seen in (20\%) of isolates. Badiee et al. (2012) reported that $30.6 \%$ of Aspergillus species isolates were resistant to ITC when tested by E-test. It was found that echinocandins exhibited a good activity against $A$. fumigatus isolates. However, azole agents had different activity in vitro. The first isolate was susceptible in vitro to ITC, VO (MIC $\leq$ $1 \mathrm{mg} / \mathrm{L}$ ), and POS (MIC value $\leq 0.25 \mathrm{mg} / \mathrm{L}$ ). The second isolate, obtained after VO therapy, was resistant in vitro to ITC, VO, and POS. Mellado et al. (2013) reported that pulmonary A. fumigatus isolates recovered from Spanish patient were multiple triazoles resistant.

Resistance to ITC is usually associated with a reduction in POS susceptibility, predictably because the 2 drugs are structurally similar (Rodriguez-Tudela et al., 2008). This study showed resistance to ITC and POS by Aspergillus section flavi. Isolates with reduced susceptibility to ITC are frequently crossresistant to other triazoles, and specific testing is recommended (Arendrup et al., 2012). The MIC results obtained by E-test methods demonstrated that POS was very active against all Aspergillus spp. (all susceptible at MIC $<0.25 \mu \mathrm{g} / \mathrm{ml}$ ). All isolates of Aspergillus spp. were inhibited with $<0.5 \mu \mathrm{g} / \mathrm{ml}$ of VO (Rudramurthy et al., 2011).

These results demonstrate the excellent efficacy of VO against Aspergillus species and suggest that VO may be the treatment of choice in invasive aspergillosis caused by $A$. fumigatus and A. flavus. VO was found to have high-MIC in 9.1\% Aspergillus isolates (Guinea et al., 2010). VO of a low-MIC was reported in $4.9 \%$ of $A$. flavus and considered as sensitive (Rudramurthy et al., 2011).

However, Kaufman et al. (2004) found that resistance of A. fumigatus to the azoles was reported to vary from a high of $52 \%$ and $38 \%$ with ITC and ravuconazole, respectively, to a low of $11 \%$ with VO. They also reported that high resistance of $A$. fumigatus to the azole agent POS was detected. 
Table 2: Susceptibility of Aspergillus isolates to the most commonly used antifungal agents by E. test.

\begin{tabular}{|c|c|c|c|}
\hline \multirow{3}{*}{ Aspergillus Species (no. of isolates) } & \multirow{3}{*}{ Antifungal agent } & \multicolumn{2}{|c|}{ MIC/MEC $(\mu \mathrm{g} / \mathrm{ml})$} \\
\hline & & \multirow{2}{*}{ Range } & E. test \\
\hline & & & $90 \%$ \\
\hline \multirow{4}{*}{ Aspergillus fumigatus (3) } & Voriconazole & $0.250-1.000$ & 0.190 \\
\hline & Itraconazole & $0.250-2.000$ & 0.500 \\
\hline & Posaconazole & $0.010-4.000$ & 4.000 \\
\hline & Caspofungin & $0.250-1.000$ & 0.125 \\
\hline \multirow{4}{*}{ Aspergillus section flavi (4) } & Voriconazole & $0.064-0.640$ & 0.250 \\
\hline & Itraconazole & $0.250-0.500$ & 0.500 \\
\hline & Posaconazole & $0.500-4.000$ & 4.000 \\
\hline & Caspofungin & $0.250-1.000$ & 0.032 \\
\hline \multirow{4}{*}{ *Aspergillus terreus (1) } & Voriconazole & $0.120-1.000$ & - \\
\hline & Itraconazole & $0.010-0.500$ & - \\
\hline & Posaconazole & $0.010-0.060$ & - \\
\hline & Caspofungin & $0.250-1.000$ & - \\
\hline \multirow{4}{*}{ Aspergillus niger (1) } & Voriconazole & $0.064-0.190$ & - \\
\hline & Itraconazole & $1.500-32.00$ & - \\
\hline & Posaconazole & 0.250 & - \\
\hline & Caspofungin & 0.032 & - \\
\hline \multirow{4}{*}{ Aspergillus candidus (1) } & Voriconazole & $0.061-0.064$ & - \\
\hline & Itraconazole & $0.064-0.250$ & - \\
\hline & Posaconazole & $0.032-0.250$ & - \\
\hline & Caspofungin & 0.032 & - \\
\hline
\end{tabular}

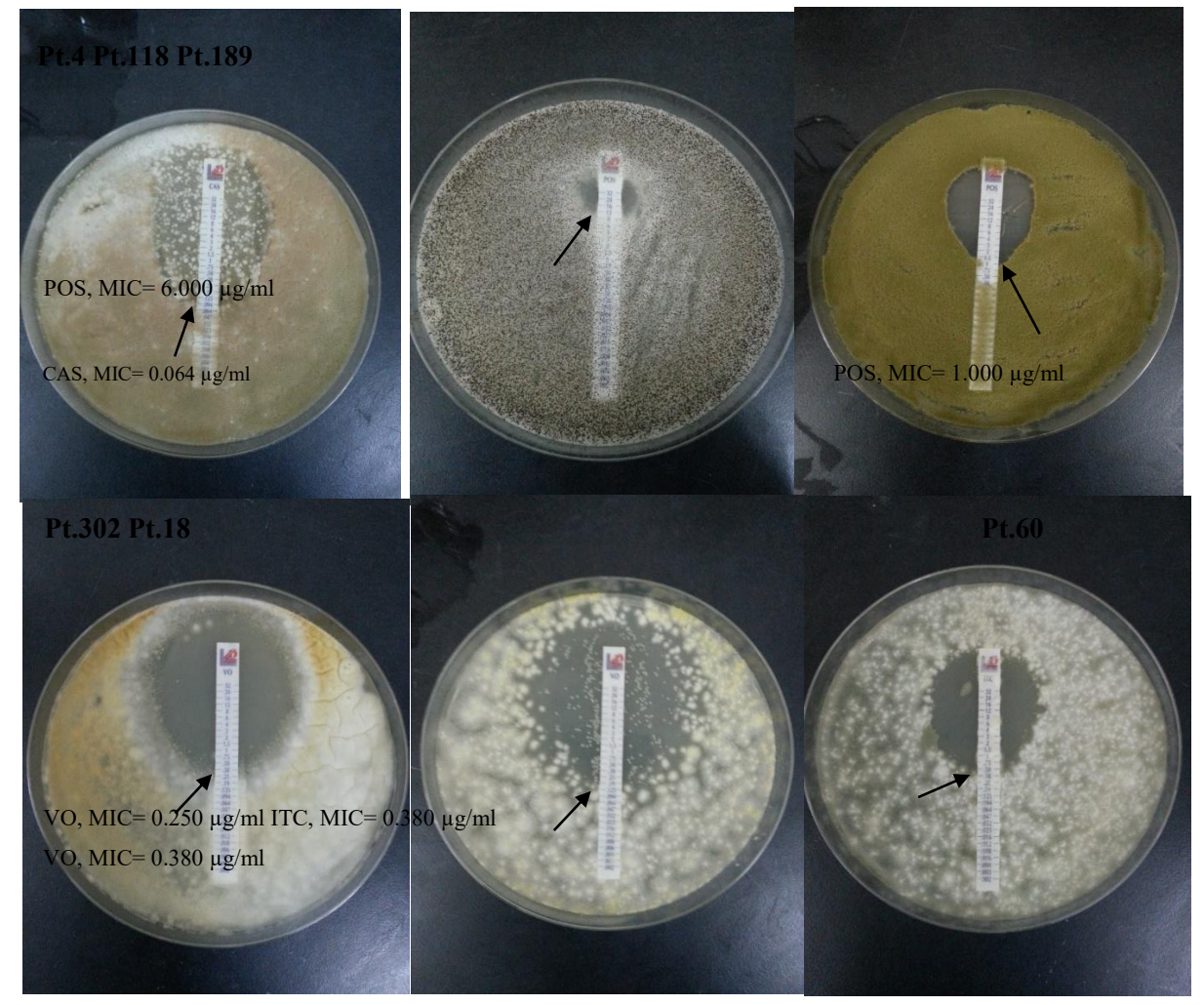

Fig. 1: E-test gradient strips of different antifungal agents and different Aspergillus species (Isolates No: 2 and 118). 


\section{CONCLUSION}

The fungus Aspergillus was found to be the predominant fungal pathogen isolated from patients with pulmonary diseases, notably from patients with COPD and pulmonary TB. Aspergillus flavus, A. niger, and A. terreus were the most frequent isolated spp. followed by A. fumigatus in the fourth place. All of the tested Aspergillus species were displaying marked resistance against traditional Azoles, (most notably to fluconazole. For the newer Azoles, VO and CAS were having full inhibitory activity against the tested Aspergilli.

\section{FINANCIAL SUPPORT AND SPONSORSHIP}

Nil.

\section{CONFLICT OF INTERESTS}

There are no conflicts of interest.

\section{REFERENCES}

Arendrup MC, Jensen RH, Grif K, Skov M, Pressler T, Johansen HK, Lass-Flörl C. In vivo emergence of Aspergillus terreus with reduced azole susceptibility and a Cyp51a M217I alteration. J. Infect. Dis. 2012; 206(6):981-985.

Aziz FZ. 2014. Isolation and Identification Aspergillus spp. isolated from the Lower Respiratory tract of people with chronic lung diseases. M.Sc thesis, College of Science, University of AL-Qadisiyia, Iraq.

Badiee P, Alborzi A, Moeini M, Haddadi P, Farshad S, Japoni A, Ziyaeyan M. Antifungal Susceptibility of the Aspergillus Species by Etest and CLSI Reference Methods. Arch. Iran Med. 2012; 15(7):429-432.

Barberan J, Alcazar B, Malmierca E, Garcia DL, Dorca J, Del $\mathrm{CD}$, Villena V, Hernandez-Febles M, Garcia-Perez FJ, Granizo JJ, Israel DA, Salama N, Arnold CN, Moss SF, Ando T, Wirth HP, Tham KT, Camorlinga M, Blaser MJ, Falkow S, Peek RM. Repeated Aspergillus isolation in respiratory samples from non-immunocompromised patients not selected based on clinical diagnoses: colonization or infection. BMC Infect. Dis. 2012; 12:295.

Brooks GF, Morse SA, Carroll KC, Mietzner TA, Butel JS. 2014. Jawetz, Melnick, \& Adelberg's Medical Microbiology. $26^{\text {th }}$ ed., Chapter 45, Med. Mycol. The McGraw-Hill Companies; United States, p. 671-713.

Buchta V, Vejsova M, Vale-Silva LA. Comparison of disk diffusion test and E-test for voriconazole and fluconazole susceptibility testing. Folia Microbiol. (Praha). 2008; 53:153-160.

Chamilos G, Kontoyiannis DP. Update on antifungal drug resistance mechanisms of Aspergillus fumigatus. Drug Resist. Update. 2005; 8(6):344-358.

Denning DW, Park S, Lass-Flörl C, Fraczek MG, Kirwan M, Gore R, Smith J, Bueid A, Moore CB, Bowyer P, Perlin DS. High-frequency triazole resistance found in nonculturable Aspergillus fumigatus from lungs of patients with chronic fungal disease. Clin. Infect. Dis. 2011; 52(9):11231129.

Diba K, Kordbacheh P, Mirhendi SH, Rezaie S, Mahmoudi M. Identification of Aspergillus species using morphological characteristics. Pak. J. Med. Sci. 2007; 23(6):867-872.

Forbes BA, Sahm FD, Weissfeld AS, Trevino EA. 2014. Mycology: Laboratory Methods in Basic Mycology. BAILEY \& SCOTT'S Diagnostic Microbiology, 13th ed. Elsevier Inc. USA, pp. 628-716.

Garnacho-Montero J, Olaechea P, Alvarez-Lerma F, AlvarezRocha L, Blanquer J, Galván B, Rodriguez A, Zaragoza R, Aguado JM, Mensa J, Solé A, Barberán J. Epidemiology, diagnosis and treatment of fungal respiratory infections in the critically ill patient. Rev. Esp. Quimioter. 2013; 26(2):173-188.

Goering RV, Dockrell HM, Zuckerman MP, Roitt IM, Chiodini PL. 2013. Mims' Medical Microbiology. $5^{\text {th }}$ ed. Chapter 19, Lower respiratory tract infections; Elsevier Inc. USA, p. 213-35.
Groll AH, Kolve H. Antifungal agents: in vitro susceptibility testing, pharmacodynamics, and prospects for combination therapy. Eur. J. Clin. Microbiol. Infect. Dis. 2004; 23(4):256-270.

Guinea J, Torres-Narbona M, Gijón P, Muñoz P, Pozo F, Peláez T, de Miguel J, Bouza E. Pulmonary aspergillosis in patients with chronic obstructive pulmonary disease: incidence, risk factors, and outcome. Clin. Microbiol. Infect. 2010; 16:870-877.

Gupta P, Khare V, Kumar D, Ahmad A, Banerjee G, Singh M. Comparative Evaluation of Disc Diffusion and E-test with Broth Microdilution in Susceptibility testing of Amphotericin B, Voriconazole and Caspofungin against Clinical Aspergillus isolates. J. Clin. Diagn. Res. 2015; 9(1):04-07.

Kaufman D, Boyle R, Hazen KC. 2004. Sensitivities of fungal isolates in high-risk preterm infants exposed to fluconazole prophylaxis in a neonatal intensive care unit over a 5-year period. Program and abstracts of the Interscience Conference on Antimicrobial Agents and Chemotherapy; Washington, DC. M-1808.

Krel M, Petraitis V, Petraitiene R, Jain MR, Zhao Y, Li H, Walsh TJ, Perlin DS. Host biomarkers of invasive pulmonary aspergillosis to monitor therapeutic response. Antimicrob. Agents Chemother. 2014; 58(6):3373-3378.

Latgé JP. The pathobiology of Aspergillus fumigatus. Trends Microbiol. 2001; 9(8):382-389.

Malik MF, Akram S, Zia R. Otomycosis; A clinico-mycological study and efficacy of tincture mertheolate in its treatment. Professional Med. J. 2009; 16(3):419-423.

Mellado E, De La Camara R, Buend B, Rodriguez-Tudela JL, Cuenca-Estrella M. Breakthrough pulmonary Aspergillus fumigatus infection with multiple triazole resistance in a Spanish patient with chronic myeloid leukemia. Rev. Iberoam. Micol. 2013; 30(1):64-68.

Mikulska M, Viscoli C. Current role of echinocandins in the management of invasive aspergillosis. Curr. Infect. Dis. Rep. 2011; 13:517-527.

Njunda AL, Ewang AA, Kamga LHF, Nsagha DS, Assob JCN, Ndah DA, Kwenti TE. Respiratory Tract Aspergillosis in the Sputum of Patients suspected of Tuberculosis in Fako division-Cameroon. J. Microbiol. Res. 2012; 2(4):68-72.

Pagano L, Fianchi L, Caira M. Pulmonary aspergillosis in hematologic malignancies: lights and shadows. Haematologica. 2008; 93:1611-1616.

Patterson TF, Kirkpatrick WR, White M, Hiemenz JW, Wingard JR, Dupont B, Rinaldi MG, Stevens DA, Graybill JR. Invasive aspergillosis. Disease spectrum, treatment practices, and outcomes. I3 Aspergillus Study Group. Med. (Baltimore). 2000; 79:250-60.

Pfaller MA, Pappas PG, Wingard JR. Invasive fungal pathogens: current epidemiological trends. Clin. Infect. Dis. 2006; 43:S3-S14.

Rodriguez-Tudela JL, Alcazar-Fuoli L, Mellado E, AlastrueyIzquierdo A, Monzon A, Cuenca-Estrella M. Epidemiological cutoffs and cross-resistance to azole drugs in Aspergillus fumigatus. Antimicrob. Agents Chemother. 2008; 52:2468-2472.

Rudramurthy SM, Geertsen E, Chakrabarti A, Mouton JW, Meis JF. In vitro susceptibility of 188 clinical and environmental isolates of Aspergillus flavus for the new triazole is a voriconazole and seven other antifungal drugs. Mycoses. 2011; 54:583-589.

Shao PL, Huang LM, Hsueh PR. Recent advances and challenges in the treatment of invasive fungal infections. Int. J. Antimicrob. Agents. 2007; 30(6):487-495.

Shujat U, Ikram A, Abbasi SA, Ayyub M, Mirza IA, Fayyaz M. Spectrum of Superficial and Deep Fungal Isolates in Northern Pakistan. Virol. Mycol. 2014; 3:131.

Singh N, Paterson DL. Aspergillus infections in transplant recipients. Clin. Microbiol. Rev. 2005; 18(1):44-69.

Snelders E, Melchers WJ, Verweij PE. Azole resistance in Aspergillus fumigatus: a new challenge in the management of invasive aspergillosis. Future Microbiol. 2011; 6(3):335-347.

Walsh TJ, Anaissie EJ, Denning DW, Herbrecht R, Kontoyiannis DP, Marr KA, Morrison VA, Segal BH, Steinbach WJ, Stevens DA, Van 
Burik JA, Wingard JR, Patterson TF. Treatment of aspergillosis: clinical practice guidelines of the Infectious Diseases Society of America. Clin. Infect. Dis. 2008; 46:327-360.
How to cite this article:

Al-Charrakh AH, Al-Mamory ZO, Al-Malaky KA. Antifungal Susceptibility Patterns of Aspergillus species isolated from patients with Pulmonary diseases in Iraq. J App Pharm Sci, 2018; 8(05): 088-093. 